\title{
Microarray analysis in pulmonary hypertension
}

\author{
Julia Hoffmann ${ }^{1}$, Jochen Wilhelm², Andrea Olschewski ${ }^{1,3}$ and \\ Grazyna Kwapiszewska ${ }^{1,3}$ \\ Affiliations: \\ ${ }^{1}$ Ludwig Boltzmann Institute for Lung Vascular Research, Graz, Austria. \\ ${ }^{2}$ Dept of Internal Medicine, Justus-Liebig-University Giessen, Universities of Giessen and Marburg Lung \\ Center, German Center for Lung Research, Giessen, Germany. \\ ${ }^{3}$ Dept of Experimental Anaesthesiology, Medical University of Graz, Graz, Austria.
}

\section{Correspondence:}

Grazyna Kwapiszewska, Ludwig Boltzmann Institute for Lung Vascular Research c/o ZMF, Stiftingtalstrasse 24, 8010 Graz, Austria.

E-mail: Grazyna.KwapiszewskaQlvr.lbg.ac.at

ABSTRACT Microarrays are a powerful and effective tool that allows the detection of genome-wide gene expression differences between controls and disease conditions. They have been broadly applied to investigate the pathobiology of diverse forms of pulmonary hypertension, namely group 1, including patients with idiopathic pulmonary arterial hypertension, and group 3, including pulmonary hypertension associated with chronic lung diseases such as chronic obstructive pulmonary disease and idiopathic pulmonary fibrosis. To date, numerous human microarray studies have been conducted to analyse global (lung homogenate samples), compartment-specific (laser capture microdissection), cell type-specific (isolated primary cells) and circulating cell (peripheral blood) expression profiles. Combined, they provide important information on development, progression and the end-stage disease. In the future, system biology approaches, expression of noncoding RNAs that regulate coding RNAs, and direct comparison between animal models and human disease might be of importance.

@ERSpublications

Comprehensive overview of compartment-specific microarray studies of material from pulmonary hypertension patients http://ow.ly/YEFO2 


\section{Introduction}

Pulmonary hypertension $(\mathrm{PH})$ includes a large spectrum of diseases with elevated pulmonary pressures $\geqslant 25 \mathrm{mmHg}$. $\mathrm{PH}$ is characterised by pulmonary vascular remodelling and associated with high resistance to blood flow in the lung, which ultimately leads to right heart failure and death [1]. PH can manifest as: pulmonary arterial hypertension (PAH) (group 1); $\mathrm{PH}$ due to left heart disease (group 2), chronic lung disease (CLD) and/or hypoxia (group 3); chronic thromboembolic PH (group 4); and PH with unclear multifactorial mechanisms (group 5) [1]. PH is a frequent (up to 60\% prevalence) and severe complication of CLD [2]. The occurrence of $\mathrm{PH}$ is an indicator of disease progression and predicts patients' outcome [2-4]. The main pathophysiological hallmark of $\mathrm{PAH}$ and $\mathrm{PH}$ with CLD is pulmonary vascular remodelling of small pulmonary arteries. This includes, most importantly, intimal hyperplasia, medial thickening due to pulmonary artery smooth muscle cell (PASMC) proliferation and, to some extent, adventitial remodelling [5, 6]. Another feature of $\mathrm{PH}$ is intra- and perivascular inflammation leading to activation of growth factor signalling pathways, and proliferation of PASMCs, which further potentiates arterial remodelling [7]. Circulating cells and their mediators have also been postulated to be involved in disease progression as they are capable of promoting recruitment, retention and differentiation of circulating monocytic cell populations that contribute to vascular remodelling $[8,9]$. Although the understanding of $\mathrm{PH}$ pathobiology has increased substantially over recent years there is still a pressing need to fully comprehend how underlying mechanisms drive vascular remodelling.

\section{RNA expression studies}

Gene expression studies, such as microarrays and RNA sequencing, provide accessible and fast screening technologies to detect genes, groups of co-regulated genes or pathways that are involved in remodelling processes. They allow for a broad and unbiased look at the differential whole-genome gene expression patterns in PH. To date, RNA expression studies have been employed to 1) identify genes and pathways that have previously not been associated with $\mathrm{PH}$ pathogenesis [10], 2) detect new potential biomarkers $[11], 3)$ identify individuals at risk for developing $\mathrm{PH}[12]$, and 4) determine the impact of medication on disease progression [13].

In addition to identifying coding RNAs (mRNA), the expression of noncoding RNAs such as microRNAs (miRNAs) can also be analysed. Unlike coding mRNAs, noncoding RNAs are not translated to proteins $[14,15]$ but can regulate expression of mRNAs at the transcriptional and post-transcriptional level [14]. Noncoding RNAs involved in epigenetic processes can be divided into two main groups: short noncoding (e.g. miRNAs <30 nt) and long noncoding RNAs (>200 nt) [16]. While short noncoding RNAs have attracted some attention in recent studies [11, 17], the information on expression, function and role of long noncoding RNAs in $\mathrm{PH}$ is still limited.

\section{Microarray technology and data analyses}

Microarray technology has been used for more than two decades, and is today well established and highly standardised on the level of instrumentation and biochemistry [18, 19]. Additionally, the majority of research uses microarrays to study gene expression. For these reasons, this review focuses on studies utilising microarray technology only.

Microarrays are tools to measure large numbers of different sequences in a complex mixture of nucleic acids. The RNA samples are amplified, labelled and hybridised to an array of spotted oligonucleotides. Image analysis identifies the spots, quantifies the signals and constructs data tables including the spot annotations that can be further processed and analysed. The data processing can include background subtraction and normalisation to adjust intensity profiles of different arrays. To identify "candidate genes" that are likely to be differentially expressed between groups or conditions, genes can be ranked by their average (logarithmically transformed) fold change or by a (possibly moderated) t-statistic, and the top-ranking genes can be identified. It is also common practice to create lists of candidate genes with a given false-discovery rate (the expected proportion of false positives among the actually rejected null hypotheses) [20]. Afterwards, genes can be analysed for co-expression patterns by clustering, multidimensional scaling and principal component analysis to identify gene sets that may be involved in similar physiological processes. Externally defined gene sets, for instance, genes belonging to a particular signalling pathway, can be analysed for statistical enrichment among the top-ranked genes (gene-set enrichment analysis) or statistical over-representation of candidate genes (over-representation analysis) [21].

\section{Challenges of microarray studies}

The difficulty in studying genes involved in pulmonary vascular remodelling may be confounded by several factors that can influence the results, such as the method of specimen acquisition and storage, RNA quality [22], pre-amplification [23], labelling protocols [24], microarray platform [25], and data analyses applied [26-29]. Additionally, the interpretation of data is prone to bias. The selection criterion is 
subjective, and a good choice depends on the context, the design and the aim of the experiment. Numerous genes are often identified as differentially regulated. Picking genes that are indeed relevant to disease pathogenesis is challenging. Mostly, the strongest differentially regulated genes are selected for further analyses. Whether these are also the genes which are biologically relevant is unpredictable. Less regulated but pathophysiologically important genes might easily be overlooked. Therefore, methods such as clustering, gene set or network analyses use the information from many genes in parallel and are thus much more robust against chance variation of individual genes. The integration of additional, external knowledge about the biology of the genes can generate hypotheses about relevant biological processes that are mirrored by the changes of the expression profiles.

The relevance of differences in gene expression detected by microarray analyses can only be estimated when combining microarray results with validation on protein level by western blotting or flow cytometry. The confirmation of findings on protein level is crucial as deregulated genes do not automatically lead to changes on protein level due to control of the proteome fingerprint by miRNAs and noncoding RNAs.

\section{Selection criteria}

This review attempts to summarise the current and relevant literature focusing on microarray based high-throughput transcriptome analyses of idiopathic PAH (IPAH) (as a prototype disease), familial pulmonary arterial hypertension (FPAH) and $\mathrm{PH}$ associated with CLD such as chronic obstructive pulmonary disease (COPD) and idiopathic pulmonary fibrosis (IPF). Human microarray studies on $\mathrm{PH}$ groups 2, 4 and 5 are limited [30], and are not included in this review. As microarray data can be derived from the analyses of different compartments of the lung, we have summarised the current status of data collected by microarray analyses of human lung homogenate samples, laser capture microdissected pulmonary arteries, and isolated or circulating cells (table 1).

The selection criteria for studies included in this review were article type (research articles published until November 2015), species (human), technology (microarray or gene array) and disease (group 1 and $3 \mathrm{PH}$ or $\mathrm{PAH}$ ). A short synopsis of rodent $\mathrm{PH}$ model microarray studies is provided at the end of this review.

\section{Microarray studies on human lung tissue}

Explanted lungs (representing the end-stage disease) or biopsy material (representing a point in time during disease progression but usually not clinically indicated and, therefore, rarely available) provide a unique opportunity to identify molecular mechanisms and potentially new therapeutic targets in the diseased lung. Although the access to human explants is rather limited, it is an even greater challenge to obtain the necessary healthy donor material for comparison. The source of control tissue can originate from downsizing of transplanted lungs, nontransplanted lungs or resection of tumours. This can bias the results of microarray analyses. Similarly, other confounding factors such as the severity of the disease, comorbidities, medication and the age/sex of the cohort might influence study outcome.

The easiest but least refined approach when analysing gene expression in $\mathrm{PH}$ is the analysis of samples from total lung homogenate. As intrapulmonary arteries represent only a minor portion of lung tissue $(<10 \%)$, the expression profile of this compartment may be largely masked or even lost when analysing lung homogenates [50]. Inevitably, the use of whole tissue results in the average of the various expression profiles coming from diverse cell types. Additionally, lung sections may contain vessels of different sizes and may have differing cell composition, which makes it difficult to interpret the results with regard to relevant biological processes. Furthermore, severe parenchyma remodelling in CLD may disguise relevant findings. Nevertheless, utilisation of lung homogenate can deliver insights on gene expression that, by further immunohistochemical validation, can lead to discovery of new potential targets involved in vascular remodelling. In order to specifically analyse gene expression in the pulmonary vasculature, several studies have applied laser capture microdissection (LCM) to isolate small pulmonary arteries [51,52]. Another approach to overcome tissue heterogeneity is to analyse isolated primary human cells such as pulmonary artery endothelial cells, PASMCs or fibroblasts. However, here, several aspects have to be taken into consideration as changes in gene expression can be affected by isolation and cell culture conditions. The impact of passaging cultured cells on gene expression is well known [53-55]. Growth factors in the culture medium can alter paracrine and hormone signalling and cell growth, and thereby can influence gene expression [56, 57]. Finally, analysis of circulating cells can provide a useful tool to detect novel biomarkers of disease progression or monitor personalised medication. Due to their clinical accessibility, circulating blood cells represent the most convenient cell source for the assessment of alteration in gene expression in $\mathrm{PH}$.

\section{Lung homogenate samples}

Using human lung homogenate samples of IPAH and FPAH, GerACi et al. [31] revealed decreased expression of genes encoding kinases and phosphatases, and upregulation of oncogenes and genes coding 
TABLE 1 Details of microarray studies published on lung homogenate (LH), laser capture microdissection (LCM), and isolated or circulating cells since 2001 with a focus on pulmonary arterial hypertension (PAH) or pulmonary hypertension (PH) associated with chronic lung diseases, displayed in chronological order

\begin{tabular}{|c|c|c|c|c|c|c|c|c|c|c|}
\hline & Patient cohort & Mean age years & $\begin{array}{c}\text { Mean mPAP } \\
\mathrm{mmHg}\end{array}$ & Biological material & $\begin{array}{c}\text { Target } \\
\text { molecule }\end{array}$ & Platform & Prominent genes & $\begin{array}{c}\text { Prominent } \\
\text { pathways/processes }\end{array}$ & $\begin{array}{l}\text { First author } \\
\text { [ref.] }\end{array}$ & GEO link \\
\hline \multirow[t]{5}{*}{ LH samples } & $\begin{array}{c}6 \text { controls (with the primary diagnosis of } \\
\text { cervical carcinoma, adenocarcinoma of the } \\
\text { lung, unknown primary diagnosis, head } \\
\text { trauma, synovial cell carcinoma and lung } \\
\text { carcinoma) } \\
6 \text { PPH subjects (2 FPPH) }\end{array}$ & NA & 59 & LH, total RNA & mRNA & Affymetrix & $\begin{array}{c}\text { TGFBR3, BMP2, MAPKK5, } \\
\text { RACK1, APOC3, LAMR1/RPSA }\end{array}$ & $\begin{array}{l}\text { Protein synthesis and } \\
\quad \text { degradation } \\
\text { Endothelial cell biology } \\
\text { Cell growth and apoptosis }\end{array}$ & GeRACI [31] & NA \\
\hline & $\begin{array}{l}13 \text { normal controls } \\
18 \text { PAH subjects } \\
8 \text { IPF subjects with } \\
\text { secondary PH }\end{array}$ & $\begin{array}{l}60 \\
44 \\
60\end{array}$ & $\begin{array}{l}55 \\
45\end{array}$ & LH, total RNA & mRNA & Agilent" & $\begin{array}{c}\text { BMPR1A, BMPR2, PTGS2, } \\
\text { VEGFA, ADAMTS9, CHL1, CYBB/ } \\
\text { NOX2, E2F1, ESR1, F2RL3, } \\
\text { MYBL1, NCOA2, P2RY1, PF4, } \\
\text { PLN, PPP2CA, TMOD3 }\end{array}$ & $\begin{array}{l}\text { Regulation of actin-based } \\
\text { motility by Rho } \\
\text { cAMP-mediated signalling } \\
\text { Protein ubiquitination } \\
\text { pathway }\end{array}$ & RAJKUMAR [32] & GSE15197 \\
\hline & $\begin{array}{l}9 \text { normal controls (normal lung tissue } \\
\text { specimens were obtained from organ donors } \\
\text { whose lungs were not used for lung } \\
\text { transplantation) } \\
9 \text { SSc-PF subjects } \\
9 \text { SSc-PAH subjects } \\
10 \text { IPF subjects } \\
8 \text { IPAH subjects }\end{array}$ & $\begin{array}{l}49 \\
52 \\
62 \\
35\end{array}$ & $\begin{array}{l}20 \\
48 \\
23 \\
60\end{array}$ & LH, total RNA & mRNA & Illumina ${ }^{+}$ & $\begin{array}{c}\text { CXCL10, FCN3, IGFBP7, } \\
\text { SCGB1A1 }\end{array}$ & $\begin{array}{l}\text { Fibrosis } \\
\text { IGF signalling } \\
\text { caveolin-mediated } \\
\text { endocytosis } \\
\text { antigen presentation } \\
\text { chemokine activity } \\
\text { IL-17 signalling }\end{array}$ & Hsu [33] & GSE48149 \\
\hline & $\begin{array}{c}17 \text { PH-IPF subjects } \\
45 \text { intermediate PH-IPF subjects } \\
22 \text { IPF subjects } \\
7 \text { IPAH subjects }\end{array}$ & $\begin{array}{l}58 \\
59 \\
61 \\
43\end{array}$ & $\begin{array}{l}48 \\
29 \\
17 \\
53\end{array}$ & LH, total RNA & mRNA & Affymetrix & $\begin{array}{l}\text { SPP1, MMP1, MMPT, MMP13, } \\
\text { EDNRB, PTX3, S100A8 }\end{array}$ & $\begin{array}{c}\text { PH phenotype: } \\
\text { cell proliferation, fibroblast } \\
\text { migration } \\
\text { noPH phenotype: } \\
\text { proinflammatory genes }\end{array}$ & MURA [12] & GSE24988 \\
\hline & $\begin{array}{c}8 \text { controls } \\
8 \text { PAH subjects }\end{array}$ & $\begin{array}{l}47 \\
40\end{array}$ & NA & LH, total RNA & mRNA & Affymetrix & СYР7B1 & Bile acids metabolites & Zнао [34] & GSE53408 \\
\hline \multirow[t]{3}{*}{ LCM } & $\begin{array}{c}6 \text { donors (nontransplanted donor lung tissue } \\
\text { showing no evidence of vascular pathology) } \\
6 \text { IPAH subjects }\end{array}$ & $\begin{array}{l}47 \\
28\end{array}$ & 102 & PAs $<500 \mu \mathrm{m}$, total RNA & mRNA & Agilent & $\begin{array}{l}\text { DAAM1, RAC1, RHOA, } \\
\text { ROCK, DSV, WNT11 }\end{array}$ & $\begin{array}{l}\text { Extracellular matrix and } \\
\text { cytoskeleton } \\
\text { Immune reaction } \\
\text { WNT signaling pathway }\end{array}$ & $\begin{array}{c}\text { Laumanns } \\
{[35]}\end{array}$ & GSE10704 \\
\hline & $\begin{array}{c}7 \text { donors (4 unused donor lung shavings and } \\
3 \text { normal lung sections obtained during } \\
\text { resection of pulmonary carcinoid tumours) } \\
8 \text { PH-IPF subjects } \\
8 \text { non-PH-IPF subjects }\end{array}$ & $\begin{array}{l}59 \\
63\end{array}$ & $\begin{array}{l}29 \\
12\end{array}$ & $\begin{array}{c}\text { PAs } 100-450 \mu \mathrm{m} \text {, total } \\
\text { RNA }\end{array}$ & mRNA & Affymetrix & STAT1, SMOC2, SFRP2 & $\begin{array}{l}\text { Amino acid metabolism } \\
\text { AMP kinase signalling } \\
\text { Ciliary neutrophic factor } \\
\text { signalling } \\
\text { Complement system } \\
\text { Cellular growth/proliferation }\end{array}$ & Рatel [36] & NA \\
\hline & $\begin{array}{l}8 \text { donors (downsized nontumorous } \\
\text { nontransplanted donor lungs) } \\
8 \text { PH-IPF subjects } \\
8 \text { PH-COPD subjects }\end{array}$ & $\begin{array}{l}44 \\
62 \\
57\end{array}$ & $\begin{array}{l}55 \\
53\end{array}$ & PAs $<300 \mu \mathrm{m}$, total RNA & mRNA & Agilent & $\begin{array}{c}\text { COLZA1, TNC, COLGA3, } \\
\text { THBS2, VWF }\end{array}$ & $\begin{array}{c}\text { Retinol metabolism } \\
\text { ECM receptor interaction }\end{array}$ & HofFmann [6] & NA \\
\hline Isolated cells & $\begin{array}{l}2 \text { normal controls } \\
2 \text { IPAH subjects }\end{array}$ & $\begin{array}{l}\text { NA } \\
44\end{array}$ & 52 & $\begin{array}{l}\text { PASMCs treated with } \\
\text { BMP-2, } 200 \mathrm{nM} \text { for } 24 \mathrm{~h} \text {; } \\
\text { passages 5-6 }\end{array}$ & mRNA & Affymetrix & $\begin{array}{c}\text { CAV2, MYCBP, FOS, ANXA5, } \\
\text { CYC1, GATA2, TGFBR1, } \\
\text { TGFBR2A, GADD34, HTR2B, F2R, } \\
\text { ITPR1 }\end{array}$ & $\begin{array}{c}\text { Cell growth and contraction } \\
\text { Apoptosis }\end{array}$ & FantozzI [37] & GSE2559 \\
\hline
\end{tabular}




\begin{tabular}{|c|c|c|c|c|c|c|c|c|c|c|}
\hline & Patient cohort & Mean age years & $\begin{array}{l}\text { Mean mPAP } \\
\text { mmHg }\end{array}$ & Biological material & $\begin{array}{c}\text { Target } \\
\text { molecule }\end{array}$ & Platform & Prominent genes & $\begin{array}{c}\text { Prominent } \\
\text { pathways/processes }\end{array}$ & $\begin{array}{l}\text { First author } \\
\text { [ref.] }\end{array}$ & GEO link \\
\hline & 3 normal controls & NA & & $\begin{array}{c}\text { HMVEC-L and HMVEC-C } \\
\text { treated with } 3,24 \text { or } \\
48 \mathrm{~h} \text { hypoxia; passages } \\
6-7\end{array}$ & mRNA & Affymetrix & $\begin{array}{c}\text { GREM1, LIMCH1, SLM2, SPON1, } \\
\text { ENPP2, PRKY, MPEG1, TM2D1, } \\
\text { CXCRT, MYO1B, BDNF }\end{array}$ & & CosteцLo [38] & GSE11341 \\
\hline & $\begin{array}{l}9 \text { normal controls } \\
9 \text { SSc-PF subjects } \\
9 \text { SSc-PAH subjects } \\
10 \text { IPF subjects } \\
6 \text { IPAH subjects }\end{array}$ & $\begin{array}{l}53 \\
49 \\
52 \\
62 \\
35\end{array}$ & $\begin{array}{l}20 \\
48 \\
23 \\
60\end{array}$ & $\begin{array}{l}\text { Fibroblasts, passage 3; } \\
\text { total RNA }\end{array}$ & mRNA & Illumina & PODN, LOX, FHL2 & $\begin{array}{c}\text { Fibrosis } \\
\text { IGF signalling } \\
\text { Caveolin-mediated } \\
\text { endocytosis } \\
\text { Antigen presentation } \\
\text { Chemokine activity } \\
\text { Interleukin-17 signalling }\end{array}$ & Hsu [33] & GSE48149 \\
\hline & 3 normal controls & NA & & $\begin{array}{c}\text { Primary HPASMCs } \\
\text { treated with } 24 \text { and } 48 \mathrm{~h} \\
\text { hypoxia; passages } 6 \text { and } \\
\text { 8; miRNA }\end{array}$ & miRNA & Exiqon§ $\$$ & $\operatorname{miR}-210$ & & Gou [39] & NA \\
\hline & $\begin{array}{c}3 \text { controls (no history of pulmonary or } \\
\text { cardiac disease or symptoms) } \\
3 \text { IPAH subjects } \\
3 \text { HPAH subjects }\end{array}$ & $\begin{array}{l}\text { NA } \\
\text { NA } \\
\text { NA }\end{array}$ & $\begin{array}{l}\text { NA } \\
\text { NA }\end{array}$ & $\begin{array}{l}\text { HPASMCs up to passage } \\
\text { 6, total RNA }\end{array}$ & mRNA & Affymetrix & $\begin{array}{l}\text { BKB2R, PLK1, PLK4, ANLN, } \\
\text { ANGPT1, DAPK1, SOD3 }\end{array}$ & $\begin{array}{c}\text { Cellular growth/proliferation/ } \\
\text { maintenance/movement Cell } \\
\text { cycle/death/survival }\end{array}$ & Yu [40] & NA \\
\hline \multirow[t]{7}{*}{ Circulating cells } & $\begin{array}{l}6 \text { normal controls } \\
7 \text { IPAH subjects } \\
8 \text { sPAH subjects }\end{array}$ & $\begin{array}{l}39 \\
43 \\
57\end{array}$ & $\begin{array}{l}60 \\
50\end{array}$ & PBMCs, total RNA & mRNA & Affymetrix & ECGF1, ADM, HVEM & $\begin{array}{l}\text { Inflammatory response } \\
\text { Response to stress } \\
\text { Cytochrome C oxidase } \\
\text { Lysosome } \\
\text { Intracellular signalling } \\
\text { cascade }\end{array}$ & BuLl [41]. & GSE703 \\
\hline & $\begin{array}{l}5 \text { healthy Caucasian controls } \\
5 \text { severe IPAH subjects }\end{array}$ & $\begin{array}{l}47 \\
46\end{array}$ & 51 & PBMCs, total RNA & mRNA & Affymetrix & DEFA1, DEFA3, S100P, PROK2 & $\begin{array}{l}\text { Inflammation } \\
\text { Immune responses }\end{array}$ & ULRICH [42] & NA \\
\hline & $\begin{array}{l}5 \text { healthy controls } \\
9 \text { IPAH subjects } \\
10 \text { PAH-SSc }\end{array}$ & $\begin{array}{l}34 \\
49 \\
61\end{array}$ & $\begin{array}{l}50 \\
51\end{array}$ & PBMCs, total RNA & mRNA & Affymetrix & MMPQ, VEGF, EREG, IL8 & $\begin{array}{l}\text { Angiogenesis } \\
\text { Chemotaxis } \\
\text { Inflammation }\end{array}$ & GRIGORYEV [43] & NA \\
\hline & $\begin{array}{l}3 \text { unaffected BMPR2 mutation carriers } \\
4 \text { affected } B M P R 2 \text { mutation carriers }\end{array}$ & $\begin{array}{l}73 \\
22\end{array}$ & $\begin{array}{l}\text { NA } \\
\text { NA }\end{array}$ & $\begin{array}{l}\text { Cultured lymphocytes, } \\
\text { total RNA }\end{array}$ & mRNA & Affymetrix & CYP1B1 & $\begin{array}{l}\text { Stress response } \\
\text { Actin organisation } \\
\text { Proliferation } \\
\text { Ras-related G-proteins } \\
\text { Calcium balance }\end{array}$ & WEST [44] & GSE10767 \\
\hline & $\begin{array}{l}10 \text { healthy controls } \\
21 \text { ISSc subjects without PAH } \\
15 \text { ISSc-PAH subjects }\end{array}$ & $\begin{array}{l}\text { NA } \\
55 \\
70\end{array}$ & $\begin{array}{l}19 \\
47\end{array}$ & PBMCs, total RNA & mRNA & Agilent & $\begin{array}{l}\text { ICAM1, IFNGR1, IL1B, IL13RA1, } \\
\text { JAK2, AIF1, CCR1, ALAS2, TIMP2 }\end{array}$ & $\begin{array}{c}\text { Vascular injury } \\
\text { Proliferation } \\
\text { Inflammatory responses }\end{array}$ & $\begin{array}{l}\text { PendergaAss } \\
\text { [45] }\end{array}$ & GSE19617 \\
\hline & $\begin{array}{c}10 \mathrm{SSc} \\
10 \mathrm{SSc}-\mathrm{PAH}\end{array}$ & $\begin{array}{l}51 \\
62\end{array}$ & $\begin{array}{l}\text { NA } \\
80\end{array}$ & PBMCs, total RNA & mRNA & Affymetrix & $I L 7 R$ & Inflammation Immunity & Risbano [46] & GSE22356 \\
\hline & $\begin{array}{l}41 \text { healthy controls } \\
30 \text { IPAH subjects } \\
19 \text { SSc subjects } \\
42 \text { SSc-PAH subjects } \\
8 \text { SSc-PH-ILD subjects }\end{array}$ & $\begin{array}{l}45 \\
52 \\
55 \\
60 \\
61\end{array}$ & $\begin{array}{l}\text { NA } \\
\text { NA } \\
\text { NA } \\
\text { NA }\end{array}$ & PBMCs, total RNA & mRNA & Illumina & ALAS2, ERAF/AHSP & Erythrocyte maturation & Cheade [47] & GSE33463 \\
\hline
\end{tabular}




\begin{tabular}{|c|c|c|c|c|c|c|c|c|c|}
\hline Patient cohort & Mean age years & $\begin{array}{l}\text { Mean mPAP } \\
\mathrm{mmHg}\end{array}$ & Biological material & $\begin{array}{c}\text { Target } \\
\text { molecule }\end{array}$ & Platform & Prominent genes & $\begin{array}{c}\text { Prominent } \\
\text { pathways/processes }\end{array}$ & $\begin{array}{l}\text { First author } \\
\text { [ref.] }\end{array}$ & GEO link \\
\hline $\begin{array}{l}8 \text { healthy controls } \\
8 \text { treatment-naive PAH subjects }\end{array}$ & $\begin{array}{l}40 \\
40\end{array}$ & 57 & Plasma, miRNA & miRNA & Geniom $^{f}$ & $\operatorname{miR}-150$ & & RHooes [11] & NA \\
\hline $\begin{array}{c}8 \text { healthy controls } \\
15 \text { moderate } \mathrm{PH} \text { subjects } \\
17 \text { severe } \mathrm{PH}\end{array}$ & $\begin{array}{l}\text { NA } \\
\text { NA } \\
\text { NA }\end{array}$ & $\begin{array}{l}17 \\
29 \\
46\end{array}$ & Plasma, miRNA & miRNA & NA & $\begin{array}{l}\text { miR-451, miR-1246, miR-23b, } \\
\text { miR-130a, miR-191 }\end{array}$ & & WEI [17] & NA \\
\hline $\begin{array}{l}28 \text { controls } \\
13 \text { PAH subjects } \\
23 \text { CF subjects }\end{array}$ & $\begin{array}{l}43 \\
41 \\
24\end{array}$ & $\begin{array}{l}67 \\
\text { NA }\end{array}$ & PBMCs, total RNA & mRNA & Agilent & TCF7, IL7R & $\begin{array}{c}\text { Metabolic process } \\
\text { T-cell receptor signalling } \\
\text { pathway }\end{array}$ & CHeSNé [48] & GSE38267 \\
\hline $\begin{array}{l}22 \text { healthy controls (free from known } \\
\text { cardiovascular disease) } \\
32 \text { idiopathic and heritable VN-PAH subjects } \\
8 \text { idiopathic and heritable VR-PAH subjects }\end{array}$ & $\begin{array}{l}\text { NA } \\
37 \\
27\end{array}$ & $\begin{array}{l}60 \\
46\end{array}$ & $\begin{array}{l}\text { Blood-derived } \\
\text { lymphocyte culture, } \\
6 \text { weeks); total RNA }\end{array}$ & mRNA & Affymetrix & DSG2, RHOQ & $\begin{array}{l}\text { Cytoskeletal/Rho GTPase } \\
\text { genes } \\
\text { Cell adhesion genes } \\
\text { Transcription factors } \\
\text { Developmental pathways }\end{array}$ & Hemnes [13] & $\begin{array}{l}\text { NA (series } \\
\text { pending) }\end{array}$ \\
\hline $\begin{array}{l}10 \text { controls } \\
12 \text { IPAH subjects }\end{array}$ & $\begin{array}{l}\text { NA } \\
56\end{array}$ & 49 & Plasma, miRNA & miRNA & Affymetrix & $\operatorname{miR}-23 a$ & & SARRIION [49] & NA \\
\hline
\end{tabular}

GEO: Gene Omnibus; NA: not available; PPH: primary pulmonary hypertension; FPPH: familial primary pulmonary hypertension; mPAP: mean pulmonary artery pressure; IFP: idiopathic pulmonary fibrosis; SSc: systemic sclerosis; PF: pulmonary fibrosis; IPAH: idiopathic pulmonary arterial hypertension; COPD: chronic obstructive pulmonary disease; HPAH: heritable pulmonary arterial hypertension; SPAH: secondary pulmonary arterial hypertension; BMPR2: bone morphogenetic protein receptor II; ISSc: limited systemic sclerosis; ILD: interstitial lung disease; CF: cystic fibrosis; VN: vasodilator-nonresponsive; VR: vasodilator responsive; PA: pulmonary artery; PASMC: pulmonary artery smooth muscle cell; BMP: bone morphogenetic protein; HMVEC-L: human lung microvascular endothelial cell; HMVEC-C: human cardiac microvascular endothelial cell; HPASMC: human pulmonary smooth muscle cell; PMBC: peripheral blood mononuclear cell: miRNA: microRNA: TGFBR3: transforming growth factor- $\beta$ receptor III: BMP2: bone morphogenetic protein 2: MAPKK5: mitogen-activated protein kinase kinase 5; RACK1: receptor for activated C kinase 1; APOC3: apolipoprotein C-III; LAMR1: laminin receptor 1; RPSA: ribosomal protein SA; BMPR1A: bone morphogenetic protein receptor IA; PTGS2: prostaglandin-endoperoxide synthase 2; VEGFA: vascular endothelial growth factor A; ADAMTS9: a disintegrin-like and metalloprotease with thrombospondin type 1 motif 9; CHL1: cell adhesion molecule L1-like; E2F1: E2F transcription factor 1; CYBB: cytochrome b-245 heavy chain; NOX2: NADPH oxidase 2; ESR1: oestrogen receptor 1; F2RL3: coagulation factor II receptor-like 3; MYBL1: v-Myb avian myeloblastosis viral oncogene homologue-like 1; NCOA2: nuclear receptor coactivator 2; P2RY1: purinergic receptor P2Y, G-protein coupled, 1: PF4: platelet factor 4; PLN: phospholamban; PPP2CA: protein phosphatase 2, catalytic subunit, $\alpha$-isozyme; TMOD3: tropomodulin 3; CXCL10: interferon- $\gamma$-inducible protein 10; FCN3: ficolin 3; IGFBP7: insulin-like growth factor-binding protein 7; SCGB1A1: secretoglobin 1A1; SPP1: secreted phosphoprotein 1 losteopontin); MMP1: matrix metalloprotease 1; MMP7: matrix metalloprotease 7; MMP13: matrix metalloprotease 13; EDNRB: endothelin receptor B; PTX3: pentraxin 3; CYP7B1: cytochrome P450 7B1; DAAM1: Dishevelled-associated activator of morphogenesis 1; RAC1: Ras-related C3 botulinum toxin substrate 1; RHOA: Ras homologue A; ROCK: RHO kinase; DSV: Dishevelled; WTN11: Wingless member 11; STAT1: signal transducer and activator of transcription 1; SMOC2: SPARC-related modular calcium binding 2; SFRP2: secreted Frizzled-related protein 2; COL3A1: collagen IIIA1; TNC: tenascin C; COL6A3: collagen VIA3; THBS2: thrombospondin 2;VWF: von Willebrand factor; CAV2: caveolin 2; MYCBP: Myc binding protein; FOS: FBJ murine osteosarcoma viral oncogene homologue: ANXA5: annexin A5; CYC1: cytochrome C1; GATA2: GATA binding protein 2; TGFBR1: transforming growth factor- $\beta$ receptor I: TGFBR2A: transforming growth factor- $\beta$ receptor Il $\alpha$; GADD34: growth arrest- and DNA damage-inducible protein 34: HTR2B: 5-hydroxytryptamine receptor 2B; F2R: coagulation factor II receptor; ITPR1: inositol trisphosphate receptor 1; GREM1: Gremlin; LIMCH1: LIM and calponin homology domains 1; SLM2: Sam68-like mammalian protein 2; SPON1: spondin 1; ENPP2: ectonucleotide pyrophosphatase/ phosphodiesterase 2; PRKY: protein kinase, Y-linked, pseudogene; MPEG1: macrophage expressed 1; TM2D1: TM2 domain-containing 1; MYO1B: myosin IB; BDNF: brain-derived neurotrophic factor; PODN: podocan; LOX: lysyl oxidase; FHL2: four and a half LIM domains 2; BKB2R: bradykinin receptor B2; PLK1: Polo-like kinase 1; PLK4: Polo-like kinase 4; ANLN: anillin; ANGPT1: angiopoietin 1; DAPK1: death-associated protein kinase 1; SOD3: superoxide dismutase 3; ECGF1: endothelial growth factor 1; ADM: adrenomedullin; HVEM: herpesvirus entry mediator: DEFA1: defensin Q1: DEFA3: defensin $\alpha 3$; S100P: S100 calcium binding protein 3; PROK2: prokineticin 2; MMP9: matrix metalloprotease 9; VEGF: vascular endothelial growth factor; EREG: epiregulin; IL8: interleukin-8; CYP1B1: cytochrome P450 1B1; ICAM1: intercellular adhesion molecule 1; IFNGR1: interferon- $\gamma$ receptor I; IL1B: interleukin-13; IL13RA1: interleukin-13 receptor $\alpha 1$; JAK2: Janus kinase 2; AIF1: allograft inflammatory factor 1; ALAS2: 5'-aminolevulinate synthase 2; TIMP2: tissue inhibitor of metalloprotease 2; IL7R: interleukin-7 receptor; ERAF: erythroid differentiation associated factor; AHSP: $\alpha$-haemoglobin stabilising factor; TCF7: T-cell factor 7; DSG2: desmoglein 2; RHOQ: Ras homologue family member Q; IGF: insulin-like growth factor; GTP: guanosine triphosphate; ECM: extracellular matrix. "\#: Affymetrix Inc., Santa Clara, CA, USA; ${ }^{\Re}$ : Agilent Technologies, Santa Clara, CA, USA; $^{+}$: Illumina Inc., San Diego, CA, USA; ${ }^{\S}$ : Exiqon A/S, Vedbæk, Denmark; ${ }^{\dagger}$ : Geniom Biochip MPEA Homo sapiens (Febit Biomed GmbH, Heidelberg, Germany). 
for ion channel proteins, as compared to controls. They also identified genes distinguishing IPAH from FPAH. These included transforming growth factor- $\beta$ receptor III (TGFRB3), bone morphogenic protein 2 (BMP2), mitogen-activated protein kinase kinase 5 (MAPKK5), receptor for activated C kinase 1 (RACK1), apolipoprotein C-III (APOC3) and the gene encoding the ribosomal protein SA/laminin receptor 1 (RPSA/ LAMR1), which were only found in the samples from patients with IPAH. In another study, a specific gene signature including genes relevant to regulation of actin-based motility, protein ubiquitination, and cAMP, transforming growth factor- $\beta$, mitogen-activated protein kinase, oestrogen receptor, nitric oxide and platelet-derived growth factor (PDGF) signalling were identified in $\mathrm{PAH}$ as compared to healthy lung tissue. Importantly, bone morphogenetic protein receptor II (BMPR2) expression was downregulated, even in subjects without a mutation in this gene [32]. Both of these studies [31,32] bring to the light that not only mutations in the BMPR2 gene, which are the most prominent cause of FPAH [58], but also downregulation of $B M P R 2$ in noncarrier patients could lead to $\mathrm{PH}$ pathogenesis. Additionally, females with IPAH displayed elevated expression of oestrogen receptor 1 (ESR1). Surprisingly, gene expression profiles of lung homogenates from PH-IPF patients resembled more those from normal controls than from PAH samples, indicating lack of a general PH signature [32]. Another microarray study of PAH samples focused on the metabolic heterogeneity. The gene encoding cytochrome P450 7B1 (CYP7B1), an isozyme for bile acid synthesis, was highly expressed in the PAH lung compared to controls [34]. Hsu et al. [33] compared gene expression profiles of systemic sclerosis (SSc)-associated pulmonary fibrosis and SSc-PAH subsets to IPF and IPAH. They detected genes involved in inflammation and activation of innate immunity such as CXCL10 (interferon- $\boldsymbol{\gamma}$-inducible protein 10) as being upregulated in all groups independent of $\mathrm{PH}$ occurrence. In contrast to the aforementioned study, here, a specific $\mathrm{PAH}$ signature was detected that included genes involved in antigen presentation and chemokine activity. This signature was proportional to the degree of the PAH phenotype. Mura et al. [12] investigated IPF samples with and without co-existing $\mathrm{PH}$. The detected $\mathrm{PH}$ gene signature was predominantly related to extracellular matrix (ECM) remodelling and PASMC and fibroblast proliferation/migration, whereas the non-PH phenotype was mostly associated with proinflammatory genes. Taken together, most of the gene expression studies of lung homogenate samples from $\mathrm{PAH}$ and lung fibrosis associated with $\mathrm{PH}$ revealed a common $\mathrm{PH}$ gene expression signature that included genes involved in cell proliferation, inflammation, immunity and ECM remodelling. The genes involved in inflammation are associated with $\mathrm{PH}$; however, their involvement has equally been shown in non-PH samples, revealing a global influence of inflammatory genes rather than a $\mathrm{PH}$ signature.

Laser capture microdissected pulmonary arteries

In 2009, LAUMANNs et al. [35] performed the first transcriptome-wide expression profiling of LCM resistance pulmonary vessels derived from explanted IPAH and nontransplanted donor lung tissues. The authors found elevated expression of planar cell polarity mediators such as Wingless member 11 (WNT11), Dishevelled (DSV) and RHO kinase (ROCK). This study pointed towards activation of developmental WNT signalling pathway in remodelling of intrapulmonary vessels. PATEL et al. [36] compared the gene expression of pulmonary arteries of IPF patients with and without coexisting $\mathrm{PH}$ to those of healthy donors, and revealed that mediators of PASMC and endothelial cell proliferation, WNT signalling, complement system activation and apoptosis were differentially expressed in IPF arterioles. The gene expression profiles of IPF and PH-IPF were similar, indicating comparable vascular changes in the IPF patient cohort with and without apparent $\mathrm{PH}$. This finding could prompt the speculation that reprogramming of the transcriptome occurs before the appearance of $\mathrm{PH}$. Comparison of gene expression profiles in intrapulmonary arteries of IPF and COPD with PH revealed that several genes belonging to the retinol metabolism and ECM pathways distinguishes the vascular remodelling of these two pulmonary diseases with $\mathrm{PH}$ [6]. Taken together, recent studies using LCM pulmonary arteries demonstrated that many identified genes were involved in WNT signalling, proliferation or ECM remodelling. LCM approaches highlighted that developmental signalling pathways are reactivated during vascular remodelling and that they could coordinate proliferative and ECM deposition processes.

\section{Isolated primary cells}

To date, several studies have investigated the mechanisms of proliferation, migration and contribution of single cell types to remodelling of the pulmonary artery wall. However, the molecular drivers of these processes remain largely unknown.

Even though endothelial dysfunction is a key feature in the pathogenesis of PAH [59], only one study so far analysed gene expression in human microvascular endothelial cells (HMVECs) by microarray analysis. Costello et al. [38] compared gene expression profiles of primary HMVECs derived from lung tissue with those of cardiac origin and analysed gene expression upon hypoxic exposure. 90 genes were identified as being differentially regulated in the lung endothelium. Gremlin (GREM1) and CXCR7 were verified as specifically upregulated in the lung cells in response to hypoxia. As GREM1 is a bone morphogenetic 
antagonist [60], this study pointed towards a regulatory relevance of $B M P R 2$ and the importance of this axis in $\mathrm{PH}$. The identification of CXCR7 emphasises the contribution of chemokines and their receptors to development of $\mathrm{PH}$ [38]. Currently, microarray studies comparing the gene expression profiles of healthy HMVECs and PH HMVECs are lacking. Therefore, the additional analysis of endothelial cells is necessary to give insight into gene expression underlying the observed endothelial dysfunction in $\mathrm{PH}$. In line with this notion, in a recent study, RHODES et al. [61] investigated endothelial transcriptomes of healthy and IPAH patients using RNA sequencing, which presents an alternative or complementary approach to microarray analysis. A novel relationship between BMPR2 dysfunction and reduced expression of endothelial collagen IV (COL4) and ephrin A1 (EFNA1) is proposed, which may underlie endothelial injury in PAH.

In contrast to endothelial cells, several studies have investigated gene expression profiles of human PASMCs. These cells constitute the primary cell type of the medial layer of the pulmonary vascular wall and are thought to contribute significantly to remodelling of the pulmonary artery. Yu et al. [40] compared gene expression patterns in human PASMCs from IPAH and hereditary PAH (HPAH) to those of controls. Differentially expressed genes showed similar trends of expression in both HPAH and IPAH PASMCs. Many genes were involved in cellular growth/proliferation and regulation of the cell cycle. Additionally, certain vasoactive receptors such as bradykinin receptor B2 $(B K B 2 R)$ were downregulated in both HPAH and IPAH cells. Apart from coding RNAs, noncoding RNAs have received increasing recognition but their involvement in remodelling processes is still elusive. In a recent study, Gou et al. [39] analysed miRNA gene expression in PASMCs and identified antiapoptotic miR-210 as the predominant miRNA induced by hypoxia. FANTOZZ et al. [37] examined whether BMP2 differentially regulates gene expression in PASMCs from normal subjects and IPAH patients. Exogenous administration of bone morphogenetic protein (BMP) might compensate for dysfunction of BMP signalling due to mutations in and/or downregulation of BMP receptors in IPAH patients. They observed that $>1000$ genes were oppositely regulated in IPAH PASMCs. The genes upregulated in IPAH cells included those associated with growth factors and ligands, membrane receptors, signal transduction proteins and kinases, transcription factors (e.g. c-Fos, c-Myc binding protein and nuclear factor $\kappa \mathrm{B}$ ), and enzymes (e.g. leukotriene C4 synthetase and ATP synthase). The genes downregulated in IPAH cells were apoptotic inducers or proapoptotic factors, membrane receptors, ion channels or transporters, transcription factors (e.g. SKI, transcription factors $\mathrm{E} 2 \mathrm{~F} 1$ and $\mathrm{TFE} 3$, and breast cancer transcription factor ZABC1), and cytoplasmic enzymes and kinases. In summary, the regulation of growth and transcription factors point towards the involvement of PASMC proliferation, which leads to medial thickening and vascular remodelling driving aggravation and acceleration of the disease.

The most outer layer of the pulmonary artery (adventitia) is composed of fibroblasts [62] and inflammatory cells [63]. The contribution of fibroblasts to vascular remodelling is controversial [62, 64]. Although they proliferate upon diverse stimuli such as PDGF-BB [65] or hypoxia [66], the thickness of adventitia in pulmonary arteries from $\mathrm{PH}$ patients is not substantially different from that of vessels from healthy subjects $[6,62]$. There is currently a very limited number of studies dealing with genes being regulated in the adventitial layer of PH. Hsu et al. [33] compared gene expression not only in lung homogenates of IPAH and SSc-PAH but also in isolated primary fibroblasts from these patients. Fibroblasts derived from SSc-PAH and IPAH had a common signature (24 genes); however, several genes distinguished these two entities. Genes strongly upregulated in IPAH fibroblasts included chemokines and interleukins (ILs) such as IL-6, IL-8 and IL-13 receptor. This study emphasises the importance of inflammatory components in the adventitial layer, which indicates a possible role of these cells in $\mathrm{PH}$ pathogenesis.

\section{Circulating cells}

In the last decade, numerous microarray studies have addressed mRNA and miRNA expression in circulating cells from PH patients (table 1). Except for one, all these studies analysed circulating peripheral blood mononuclear cells (PBMCs), which include multiple cell types such as lymphocytes, monocytes and natural killer cells.

In 2004, Bull et al. [41] compared gene expression of PBMCs from IPAH and secondary PAH (sPAH) (including portal hypertension, calcinosis, Raynaud's phenomenon, oesophageal dysmotility, sclerodactyly, interstitial lung disease, exposure to the anorexigens phentermine/fenfluramine and chronic pulmonary thromboembolic disease) patients with controls, and found the gene ontology classes "inflammatory response" and "response to stress" to be modulated in IPAH compared to healthy controls. IPAH and sPAH had similar expression profiles. Similarly, ULRICH et al. [42] found genes involved in inflammatory mechanisms, host defense or endothelial function were affected in IPAH PBMCs. A study comparing PBMCs from SSc-PAH to IPAH PBMCs indicated that PBMC gene expression was similar in both disease groups and correlated with known predictors of survival in PAH. This study additionally supports the notion that angiogenesis and chemotaxis/inflammation are associated with severity of PAH [43]. A study 
in 2010 by Pendergrass et al. [45] investigated whether the coexistence of PAH in limited systemic sclerosis (ISSc) influenced gene expression in PBMCs. Gene expression analysis distinguished 1SSc samples from healthy controls and separated ISSc-PAH from ISSc without PAH, pointing towards a specific PAH signature. CHEADLE et al. [47] analysed transcript profiles of PBMCs from IPAH and SSc-PAH as compared to healthy donors. Multiple gene expression signatures were found that distinguished the various disease groups from controls. One of these gene sets, erythrocyte maturation, was enriched specifically in PAH and correlated with haemodynamic measures of increasing disease severity in patients with IPAH. RisBano et al. [46] compared gene expression profiles of PBMCs from SSc patients with and without coexisting PAH, and identified IL-7 receptor (IL7R) to be significantly decreased in samples from PAH patients. CHESNé et al. [48] compared PBMC gene expression from healthy controls, PAH and cystic fibrosis patients in order to identify an end-stage chronic respiratory disease-related gene signature. Microarray results were validated in a second independent cohort and COPD patients were added to validate the common signature. In the common signature group, T-cell factor 7 (TCF7) and IL7R, two genes related to T-lymphocyte activation, were found to be downregulated. Taken together, two studies emphasise the importance of IL7R as a potential biomarker in PH. HEMNEs et al. [13] compared PBMCs from vasodilator-responsive (VR)-PAH to vasodilator-nonresponsive $\mathrm{PAH}$ patients. Differences in gene expression patterns on microarray analysis included cell-cell adhesion factors, and cytoskeletal and Rho GTPase genes. DSG2, encoding a desmosomal cadherin involved in WNT/ $\beta$-catenin signalling, and Ras homologue family member Q $(R H O Q)$, which encodes a cytoskeletal protein involved in insulin-mediated signalling, were sufficient to correctly distinguish the five VR-PAH patients in the validation cohort from the rest of the cohort, implying prognostic potential of these genes.

In circulating PBMCs, miRNA profiles also have been analysed. RHODEs et al. [11] showed that miR-150 was downregulated in the plasma of IPAH patients and correlated with the 2-year survival of these patients. Another study of PBMCs from PH patients compared healthy to moderate (mean mean pulmonary artery pressure (mPAP) $29 \mathrm{mmHg}$ ) and severe (mean mPAP $46 \mathrm{mmHg}$ ) $\mathrm{PH}$, and detected several miRNAs as being downregulated (miR-451 and miR-1246) or upregulated (miR-23b, miR-130a and miR-191), and whose expression levels were proportional to the degree of PH [17]. A recent study by SARRION et al. [49] highlighted the potential relevance of miR-23a in IPAH as it correlated with the patient's pulmonary function and might therefore serve as a potential biomarker.

To date, there has only been a single study analysing a subpopulation of PBMCs, namely lymphocytes. WEST et al. [44] generated immortalised lymphocytes from FPAH patients with BMPR2 mutations as compared to mutation positive but disease-free family members, and subjected the resulting lymphoblastoid cell lines to microarray analysis. Disease status in BMPR2 mutation carriers correlated with differential gene expression in proliferation, GTP signalling and stress response pathways. The oestrogen metabolism gene CYP1B1 (cytochrome P450 1B1) was found to be significantly downregulated in female PAH patients. The low expression of this gene was associated with disease severity. Importantly, this study illustrates that BMPR2 mutations influence inflammatory cell gene expression profiles. In the future, further microarray analyses of PBMC subpopulations should be conducted to get deeper insight into alterations of gene profiles in specific populations and to delineate their individual contribution to $\mathrm{PH}$. Additionally, expression profiles of other cell types found in the blood, such as polymorphonuclear neutrophils, have not been analysed so far. This may, however, be of relevance, as the neutrophil to lymphocyte ratio is reported to be significantly increased in patients with PAH [67].

Taken together, the easy accessibility and identification of several proposed circulating markers such as miRNAs (particularly miR-23) provide an opportunity to apply them as prognostic tools for IPAH or PH associated with lung diseases and determination of disease progression or therapy evaluation. However, detected differentially regulated gene signatures in disease conditions have to be validated in a separate cohort to confirm prognostic potential. This has so far only been performed in a very limited number of studies. Experience from cancer research has demonstrated how essential such verifications are for the approval of reliable biomarkers [68].

\section{Summary of microarray studies on rodent lung tissue}

In parallel to studies on human samples, several microarray studies have been performed on hypoxia- and monocrotaline (MCT)-induced $\mathrm{PH}$ in rodents. Although animal models may recapitulate only some aspects of human vascular remodelling, such as PASMC proliferation or inflammation, they represent an excellent tool to investigate not only end-stage disease, but also disease onset, progression or reversal.

The classical and well accepted model for mild PH is hypoxia-induced PH in rats and mice [69]. Investigators have concentrated on diverse disease stages and analysed gene expression at different time-points of disease development (short hypoxia exposure [70, 71]), end-stage disease (long-term hypoxia exposure $[50,72]$ ) or reverse remodelling (reoxygenation $[73,74]$ ). Cumulatively, these studies 
revealed that during disease onset, multiple transcription and growth factors, such as endothelin (EDN1) [70] and neurotrophic tyrosine kinase receptor type 2 (NTRK2) [71], are activated. In end-stage disease, marked structural changes have been observed, such as in procollagen, tenascin C (TNC), S100 calcium-binding protein A4 (S100A4), cluster of differentiation 36 (CD36) and FK506 binding protein 1A (FKBP1A) [50]. The reverse remodelling approaches can shed the light on genes that are involved in reverse remodelling processes, which include adenosylmethionine decarboxylase 1 (AMD1) [74], Ras GTPase-activating-like protein (IQGAP1), insulin-like growth factor-binding protein 3 (IGFBP3) and lactoferrin $(L T F)$ [73].

The inflammatory component of human PH is best resembled by the MCT-induced PH model in rats [75]. In this PH model, numerous studies analysed mRNA or miRNA expression changes and revealed alterations in genes involved in response to wounding and inflammation, blood vessel morphogenesis and genes encoding proteases [76-78], as well as miR-22, miR-30, let-7f, miR-322 and miR-451, miR-21, and let-7a [79].

Additionally, diverse studies have examined the influence of $\mathrm{PH}$ medication testing [80], strain differences [81], intermittent or sustained exposure to hypoxia [82] and sex differences [83] on the gene expression changes during development of $\mathrm{PH}$ in various rodent models.

\section{Conclusions, limitations and outlook}

To date, more than 25 human microarray studies supplying enormous amounts of gene expression data, ranging from single gene expression to complex pathway analyses, have been performed. Over the last 15 years, more easily accessible compartments, such as circulating cells, have been investigated more frequently (figure 1).

Gene expression changes in the ECM, angiogenesis, inflammatory processes and the WNT pathway are now increasingly recognised to be important for processes underlying PH (table 1 and figure 2). The direct link between WNT signalling and developmental processes, cell proliferation and migration [84] points towards its contribution to vascular remodelling. Although individual fingerprints are observed for diverse cell types and compartments, many of the regulated pathways are shared. This indicates that similar signalling patterns are involved in $\mathrm{PH}$ pathogenesis in different pulmonary compartments (figure 2). Whether these are adaptive or pathological responses and what the precise mechanisms and kinetics of their regulation are has yet to be elucidated. Further understanding of the underlying mechanisms could help to develop effective treatment options for patients with PAH and PH associated with CLD.

Each of the studies discussed has concentrated on a certain aspect of disease pathogenesis. It can be envisioned that the identification of common signalling signatures and local gene regulatory networks could lead to detection of important regulators governing more fundamental biological processes and pathways or specific parts of it, which are involved in the pathogenesis of PH. Moreover, vast amounts of data remain to be explored. The lack of consistency in sample collection, storage and preparation as well as microarray platform and bioinformatic analysis hampers direct comparisons and prevents general conclusions. However, the available data can still be a valuable source for the generation of new hypotheses

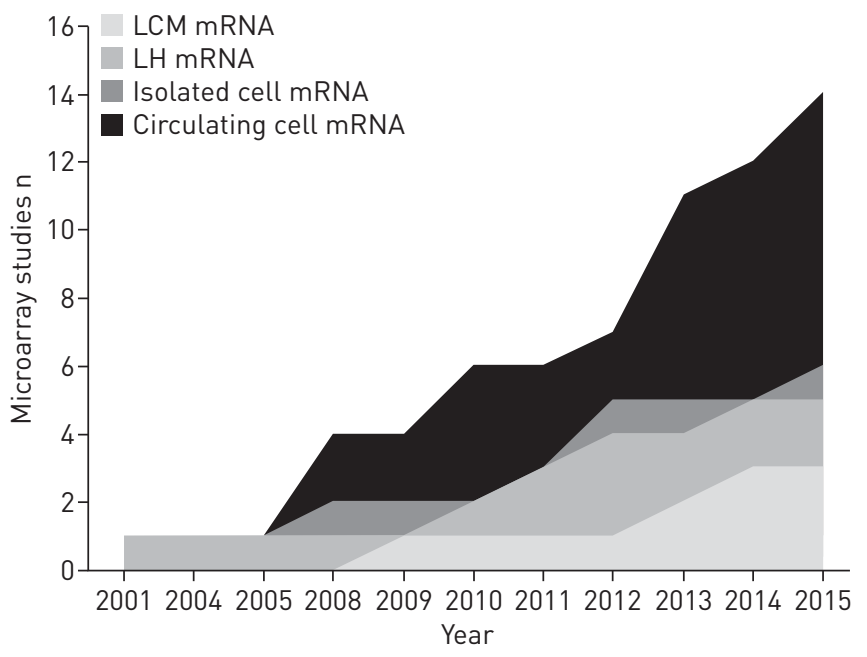

FIGURE 1 The number of human microarray studies published since 2001 on lung homogenate (LH), laser capture microdissection (LCM), and isolated or circulating cells with a focus on pulmonary arterial hypertension or pulmonary hypertension associated with chronic lung diseases. 


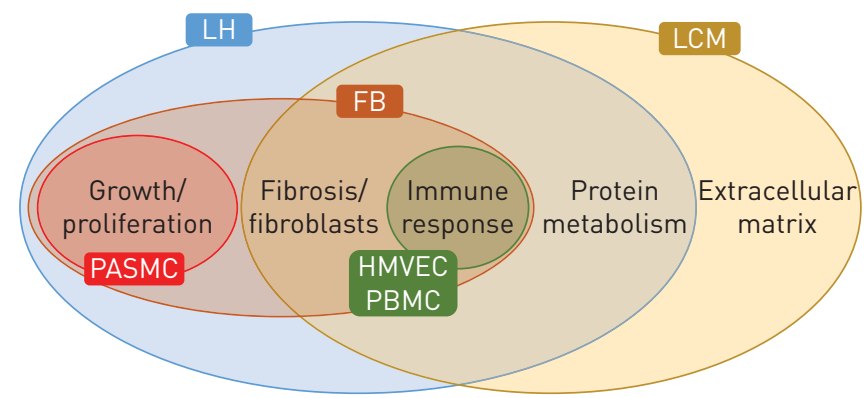

FIGURE 2 Venn diagram depicting overlapping and diverging microarray analysis results of the discussed studies. LH: lung homogenate, LCM: laser capture microdissection, FB: isolated fibroblast, PASMC: pulmonary artery smooth muscle cell; HMVEC: human microvascular endothelial cell; PBMC: peripheral blood mononuclear cell.

about the molecular mechanisms driving $\mathrm{PH}$. The utility of microarray results could be enhanced by standardisation of data acquisition and analysis, and thus hold the promise that they may significantly improve our understanding of $\mathrm{PH}$ pathomechanisms.

In the near future, new technologies will complement microarray platform. Exemplarily, next-generation RNA sequencing will be applied to reveal a snapshot of RNA presence and quantity, alternative splicing, post-transcriptional modifications, gene fusion, and mutations/single-nucleotide polymorphisms. Therefore, it will help to yield a more holistic impression of the disease pathogenesis [85]. Indeed, a recent study already utilised this technology to investigate endothelial transcriptomes of IPAH patients [61].

In summary, in future RNA expression studies, it will be important to 1) compare and harmonise gene expression from animal models and human disease, 2) use system biology approaches in order to identify complex disease development signatures and to understand the dynamics of disease development on the level of regulatory networks, and 3) analyse noncoding gene expression that could influence coding gene expression.

\section{Acknowledgement}

We would like to thank Leigh Marsh (Ludwig Boltzmann Institute for Lung Vascular Research, Graz, Austria) for critical reading of the manuscript.

\section{References}

1 Simonneau G, Gatzoulis MA, Adatia I, et al. Updated clinical classification of pulmonary hypertension. J Am Coll Cardiol 2013; 62: 25 Suppl., D34-D41.

2 Seeger W, Adir Y, Barbera JA, et al. Pulmonary hypertension in chronic lung diseases. J Am Coll Cardiol 2013; 62: 25 Suppl., D109-D116.

3 Castria D, Refini RM, Bargagli E, et al. Pulmonary hypertension in idiopathic pulmonary fibrosis: prevalence and clinical progress. Int J Immunopathol Pharmacol 2012; 25: 681-689.

4 Elwing J, Panos RJ. Pulmonary hypertension associated with COPD. Int J Chron Obstruct Pulmon Dis 2008; 3: 55-70.

5 Hoffmann J, Marsh LM, Pieper M, et al. Compartment-specific expression of collagens and their processing enzymes in intrapulmonary arteries of IPAH patients. Am J Physiol Lung Cell Mol Physiol 2015; 308: L1002-L1013.

6 Hoffmann J, Wilhelm J, Marsh LM, et al. Distinct differences in gene expression patterns in pulmonary arteries of patients with chronic obstructive pulmonary disease and idiopathic pulmonary fibrosis with pulmonary hypertension. Am J Respir Crit Care Med 2014; 190: 98-111.

7 Pullamsetti SS, Savai R, Janssen W, et al. Inflammation, immunological reaction and role of infection in pulmonary hypertension. Clin Microbiol Infect 2011; 17: 7-14.

8 Burke DL, Frid MG, Kunrath CL, et al. Sustained hypoxia promotes the development of a pulmonary artery-specific chronic inflammatory microenvironment. Am J Physiol Lung Cell Mol Physiol 2009; 297: L238-L250.

9 Foris V, Kovacs G, Tscherner M, et al. Biomarkers in pulmonary hypertension: what do we know? Chest 2013; 144: 274-283.

10 West JD, Austin ED, Gaskill C, et al. Identification of a common Wnt-associated genetic signature across multiple cell types in pulmonary arterial hypertension. Am J Physiol Cell Physiol 2014; 307: C415-C430.

11 Rhodes CJ, Wharton J, Boon RA, et al. Reduced microRNA-150 is associated with poor survival in pulmonary arterial hypertension. Am J Respir Crit Care Med 2013; 187: 294-302.

12 Mura M, Anraku M, Yun Z, et al. Gene expression profiling in the lungs of patients with pulmonary hypertension associated with pulmonary fibrosis. Chest 2012; 141: 661-673.

13 Hemnes AR, Trammell AW, Archer SL, et al. Peripheral blood signature of vasodilator-responsive pulmonary arterial hypertension. Circulation 2015; 131: 401-409.

14 Djebali S, Davis CA, Merkel A, et al. Landscape of transcription in human cells. Nature 2012; 489: 101-108. 
Neurobiol Dis 2005; 18: 649-655.

\section{Heart Circ Physiol 2010; 298: H1235-H1248.}

33 Hsu E, Shi H, Jordan RM, et al. Lung tissues in patients with systemic sclerosis have gene expression patterns unique to pulmonary fibrosis and pulmonary hypertension. Arthritis Rheum 2011; 63: 783-794.

34 Zhao YD, Yun HZ, Peng J, et al. De novo synthesize of bile acids in pulmonary arterial hypertension lung. Metabolomics 2014; 10: 1169-1175.

35 Laumanns IP, Fink L, Wilhelm J, et al. The noncanonical WNT pathway is operative in idiopathic pulmonary arterial hypertension. Am J Respir Cell Mol Biol 2009; 40: 683-691.

36 Patel NM, Kawut SM, Jelic S, et al. Pulmonary arteriole gene expression signature in idiopathic pulmonary fibrosis. Eur Respir J 2013; 41: 1324-1330.

37 Fantozzi I, Huang W, Zhang J, et al. Divergent effects of BMP-2 on gene expression in pulmonary artery smooth muscle cells from normal subjects and patients with idiopathic pulmonary arterial hypertension. Exp Lung Res 2005; 31: 783-806.

38 Costello CM, Howell K, Cahill E, et al. Lung-selective gene responses to alveolar hypoxia: potential role for the bone morphogenetic antagonist gremlin in pulmonary hypertension. Am J Physiol Lung Cell Mol Physiol 2008; 295: L272-L284.

39 Gou D, Ramchandran R, Peng X, et al. miR-210 has an antiapoptotic effect in pulmonary artery smooth muscle cells during hypoxia. Am J Physiol Lung Cell Mol Physiol 2012; 303: L682-L691.

$40 \mathrm{Yu}$ J, Wilson J, Taylor L, et al. DNA microarray and signal transduction analysis in pulmonary artery smooth muscle cells from heritable and idiopathic pulmonary arterial hypertension subjects. J Cell Biochem 2015; 116: 386-397.

41 Bull TM, Coldren CD, Moore M, et al. Gene microarray analysis of peripheral blood cells in pulmonary arterial hypertension. Am J Respir Crit Care Med 2004; 170: 911-919.

42 Ulrich S, Taraseviciene-Stewart L, Huber LC, et al. Peripheral blood B lymphocytes derived from patients with idiopathic pulmonary arterial hypertension express a different RNA pattern compared with healthy controls: a cross sectional study. Respir Res 2008; 9: 20.

43 Grigoryev DN, Mathai SC, Fisher MR, et al. Identification of candidate genes in scleroderma-related pulmonary arterial hypertension. Transl Res 2008; 151: 197-207.

44 West J, Cogan J, Geraci M, et al. Gene expression in BMPR2 mutation carriers with and without evidence of pulmonary arterial hypertension suggests pathways relevant to disease penetrance. BMC Med Genomics 2008; $1: 45$.

45 Pendergrass SA, Hayes E, Farina G, et al. Limited systemic sclerosis patients with pulmonary arterial hypertension show biomarkers of inflammation and vascular injury. PloS One 2010; 5: e12106.

46 Risbano MG, Meadows CA, Coldren CD, et al. Altered immune phenotype in peripheral blood cells of patients with scleroderma-associated pulmonary hypertension. Clin Transl Sci 2010; 3: 210-218

47 Cheadle C, Berger AE, Mathai SC, et al. Erythroid-specific transcriptional changes in PBMCs from pulmonary hypertension patients. PloS One 2012; 7: e34951.

48 Chesne J, Danger R, Botturi K, et al. Systematic analysis of blood cell transcriptome in end-stage chronic respiratory diseases. PLoS One 2014; 9: e109291.

49 Sarrion I, Milian L, Juan G, et al. Role of circulating miRNAs as biomarkers in idiopathic pulmonary arterial hypertension: possible relevance of miR-23a. Oxid Med Cell Longev 2015; 2015: 792846.

50 Kwapiszewska G, Wilhelm J, Wolff S, et al. Expression profiling of laser-microdissected intrapulmonary arteries in hypoxia-induced pulmonary hypertension. Respir Res 2005; 6: 109. 
51 Fink L, Kohlhoff S, Stein MM, et al. cDNA array hybridization after laser-assisted microdissection from nonneoplastic tissue. Am J Pathol 2002; 160: 81-90.

52 Fink L, Kwapiszewska G, Wilhelm J, et al. Laser-microdissection for cell type- and compartment-specific analyses on genomic and proteomic level. Exp Toxicol Pathol 2006; 57: Suppl. 2, 25-29.

53 Erac Y, Selli C, Filik P, et al. Effects of passage number on proliferation and store-operated calcium entry in A7r5 vascular smooth muscle cells. J Pharmacol Toxicol Methods 2014; 70: 1-5.

54 Roh M, Song C, Kim J, et al. Chromosomal instability induced by Pim-1 is passage-dependent and associated with dysregulation of cyclin B1. J Biol Chem 2005; 280: 40568-40577.

55 Sivanathan L, Chow A, Wong A, et al. In vivo passage of human prostate cancer cells in mice results in stable gene expression changes affecting numerous cancer-associated biological processes. Prostate 2014; 74: 537-546.

56 Arias ME, Ross PJ, Felmer RN. Culture medium composition affects the gene expression pattern and in vitro development potential of bovine somatic cell nuclear transfer (SCNT) embryos. Biol Res 2013; 46: 452-462.

57 Hribal R, Jewgenow K, Braun BC, et al. Influence of culture medium composition on relative mRNA abundances in domestic cat embryos. Reproduct Domest Anim 2013; 48: 245-251.

58 Machado RD, Aldred MA, James V, et al. Mutations of the TGF-beta type II receptor BMPR2 in pulmonary arterial hypertension. Hum Mutat 2006; 27: 121-132.

59 Huertas A, Perros F, Tu L, et al. Immune dysregulation and endothelial dysfunction in pulmonary arterial hypertension: a complex interplay. Circulation 2014; 129: 1332-1340.

60 Cahill E, Costello CM, Rowan SC, et al. Gremlin plays a key role in the pathogenesis of pulmonary hypertension. Circulation 2012; 125: 920-930.

61 Rhodes CJ, Im H, Cao A, et al. RNA sequencing analysis detection of a novel pathway of endothelial dysfunction in pulmonary arterial hypertension. Am J Respir Crit Care Med 2015; 192: 356-366.

62 Stacher E, Graham BB, Hunt JM, et al. Modern age pathology of pulmonary arterial hypertension. Am J Respir Crit Care Med 2012; 186: 261-272.

63 Grunig G, Durmus N. Spotlight on inflammation in pulmonary hypertension. Am J Respir Crit Care Med 2015; 192: 913-915.

64 Stenmark KR, Nozik-Grayck E, Gerasimovskaya E, et al. The adventitia: essential role in pulmonary vascular remodeling. Compr Physiol 2011; 1: 141-161.

65 Panzhinskiy E, Zawada WM, Stenmark KR, et al. Hypoxia induces unique proliferative response in adventitial fibroblasts by activating PDGF $\beta$ receptor-JNK1 signalling. Cardiovasc Res 2012; 95: 356-365.

66 Stenmark KR, Gerasimovskaya E, Nemenoff RA, et al. Hypoxic activation of adventitial fibroblasts: role in vascular remodeling. Chest 2002; 122: 6 Suppl., 326S-334S.

67 Yildiz A, Kaya H, Ertas F, et al. Association between neutrophil to lymphocyte ratio and pulmonary arterial hypertension. Turk Kardiyoloji Dernegi Arsivi 2013; 41: 604-609.

68 Vansteenkiste J, Dooms C, Mascaux C, et al. Screening and early detection of lung cancer. Ann Oncol 2012; 23: Suppl. 10, x320-x327.

69 Stenmark KR, Meyrick B, Galie N, et al. Animal models of pulmonary arterial hypertension: the hope for etiological discovery and pharmacological cure. Am J Physiol Lung Cell Mol Physiol 2009; 297: L1013-L1032.

70 Biasin V, Chwalek K, Wilhelm J, et al. Endothelin-1 driven proliferation of pulmonary arterial smooth muscle cells is c-fos dependent. Int J Biochem Cell Biol 2014; 54: 137-148.

71 Kwapiszewska G, Chwalek K, Marsh LM, et al. BDNF/TrkB signaling augments smooth muscle cell proliferation in pulmonary hypertension. Am J Pathol 2012; 181: 2018-2029.

72 Hoshikawa Y, Nana-Sinkam P, Moore MD, et al. Hypoxia induces different genes in the lungs of rats compared with mice. Physiol Genomics 2003; 12: 209-219.

73 Gharib SA, Luchtel DL, Madtes DK, et al. Global gene annotation analysis and transcriptional profiling identify key biological modules in hypoxic pulmonary hypertension. Physiol Genomics 2005; 22: 14-23.

74 Weisel FC, Kloepping C, Pichl A, et al. Impact of S-adenosylmethionine decarboxylase 1 on pulmonary vascular remodeling. Circulation 2014; 129: 1510-1523.

75 Kay JM, Harris P, Heath D. Pulmonary hypertension produced in rats by ingestion of Crotalaria spectabilis seeds. Thorax 1967; 22: 176-179.

76 Spees JL, Whitney MJ, Sullivan DE, et al. Bone marrow progenitor cells contribute to repair and remodeling of the lung and heart in a rat model of progressive pulmonary hypertension. FASEB J 2008; 22: 1226-1236.

77 van Albada ME, Bartelds B, Wijnberg $\mathrm{H}$, et al. Gene expression profile in flow-associated pulmonary arterial hypertension with neointimal lesions. Am J Physiol Lung Cell Mol Physiol 2010; 298: L483-L491.

78 Vaszar LT, Nishimura T, Storey JD, et al. Longitudinal transcriptional analysis of developing neointimal vascular occlusion and pulmonary hypertension in rats. Physiol Genomics 2004; 17: 150-156.

79 Caruso P, MacLean MR, Khanin R, et al. Dynamic changes in lung microRNA profiles during the development of pulmonary hypertension due to chronic hypoxia and monocrotaline. Arterioscler Thromb Vasc Biol 2010; 30: 716-723.

80 Moreno-Vinasco L, Gomberg-Maitland M, Maitland ML, et al. Genomic assessment of a multikinase inhibitor, sorafenib, in a rodent model of pulmonary hypertension. Physiol Genomics 2008; 33: 278-291.

81 Tada Y, Laudi S, Harral J, et al. Murine pulmonary response to chronic hypoxia is strain specific. Exp Lung Res 2008; 34: 313-323.

$82 \mathrm{Wu}$ W, Dave NB, Yu G, et al. Network analysis of temporal effects of intermittent and sustained hypoxia on rat lungs. Physiol Genomics 2008; 36: 24-34.

83 White K, Loughlin L, Maqbool Z, et al. Serotonin transporter, sex, and hypoxia: microarray analysis in the pulmonary arteries of mice identifies genes with relevance to human PAH. Physiol Genomics 2011; 43: 417-437.

84 Dejana E. The role of wnt signaling in physiological and pathological angiogenesis. Circ Res 2010; 107: 943-952.

85 Maher CA, Kumar-Sinha C, Cao X, et al. Transcriptome sequencing to detect gene fusions in cancer. Nature 2009; 458: 97-101 